\title{
Eye Tracking Evaluation Of Chinese Web Sites For The Chinese Market
}

Gabriele R. Theuner, (E-mail: gtheuner@fmarion.edu), Francis Marion Univerity, Italy

Stefan Steinmetz, (E-mail: stefansteinmetz@web.de), University of Applied Sciences, Germany

\begin{abstract}
This paper assesses and analyzes ways Chinese and European people view web sites designed in English and Chinese languages. The results suggest some similarities and differences based on different cultures.

The Chinese market is gaining more and more importance in the international business arena. Due to the strong Chinese Internet growth rates increasingly more global enterprises use the Internet for e-Commerce, market penetration and expansion, and to communicate information in China. But the success of online communication with customers in the Chinese market is in many cases not as effective as planned, because of cultural differences between Chinese and European people. Specific reading behavior, different cultural color definitions, diverse meanings of symbols or pictures as well as differing Chinese attitudes and values have to be considered when designing web sites for China.
\end{abstract}

To gain more knowledge about the needs, tastes and behavior styles from Chinese Internet users, an experiment was designed with the following hypothesis: Chinese and Europeans differ concerning web site evaluation and recognition due to culture. Web sites designed for Chinese (Mercedes, Sony Ericsson, and China Eastern) were tested using an Eye Tracking camera.

Observation, using an Eye Tracking camera, combined with a survey, showed that Chinese users share only preferences like clarity and comprehensibility in web site quality with German users. Significant differences can be named concerning amount of information, design/color design and terminology. During the tests it also became clear that the order in which the Chinese users look at elements (navigation bar, text, pictures, etc.), and the tested recognition is not identical to European users.

\section{Introduction}

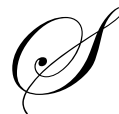

ince Chinese economy is improving rapidly, China has an enormous economic potential and is likely to have a dramatic effect on the world of e-commerce. With the acceptance of China into the World Trade Organization (WTO), great opportunities for global trade are opening up for companies eager to do business with China. The number of Chinese Internet users has exploded from 1.17 million in 1998 to over 68 million by mid 2003, giving China a top rank in international Internet usage. The steady rise in Internet users has made Chinese the most widespread language on the net behind English. Of the total world Internet population of 680 million users, 35.6\% speak English, 12.2\% Chinese, and 9.5\% Japanese. (CNNIC, Website) Since the end of the 1990's, the Chinese computer network ranked as one of the most technologically advanced in worldwide comparison. (Staiger et al, 1998, p. 331)

Bearing this in mind a great deal of western enterprises are or have already resolved to tackle the Chinese online market and use the Internet for e-commerce, market penetration, and expansion, and to communicate information in China. Because of cultural differences between China and Europe, the web presence of many enterprises is seldom as effective as planned. Possible specific reading behavior, different cultural color definitions, 
different online behavior, diverse meanings of symbols and pictures as well as differing Chinese attitudes and values have to be considered when designing web sites for China.

As companies become globally more active, multicultural studies are gaining essential importance for their business success. As a result, the differences and similarities of market characteristics must be compared for each country. (Cateora et al, 1999, pages 208-209) The East Asia culture is a main element of market characteristics. The aim of this research project is to determine fundamental directions for online communication concerning the East Asia countries, starting with China. Priorities therefore are set up in the field of web site perception and establishing a basis for Chinese specific usability tests with the help of an eye tracking device. The results should help western enterprises to improve the online communication effectiveness in East Asia. Starting with China at first it is necessary to research do's and don'ts of web site design, on the basis of the cross-cultural similarities and differences compared with common western design practice.

\section{Cultural Elements in Web Design}

It is very important to understand what the intended users, i.e. the target group, desire in order to get them involved. Web quality is based on reliability, correctness, and efficiency. Usability measures the degree of customer orientation of a web site and tailoring the web site design to a user's expectations is essential for the success of a web site.

Usability tests have determined that that navigation success depends strongly on a comprehensible site structure with an efficient search function, on clarity, and on understandable links, hyperlinks, and graphical navigation elements. Navigation problems in connection with a negative rating of the perception are usually due to a bad comprehensibility and clarity of the websites. (Theuner, 2000, pages 71-73) From the perspective of the international user, preferences for specific web sites depend not only on the usability of the web site, but also on cultural features, special user interests and individual taste.

For intercultural web design, like for the Chinese market, these web designers should keep in mind:

1. It is essential to first understand one's own culture before dealing with others.

2. It is also important to consider that Chinese think, feel and act differently in many situations than Europeans (high context/low context cultures).

3. The user behavior might be the reason for essential differences between Chinese and Latin based languages. Today, the Chinese script consists of characters that range from simple pictographic representations of objects, to complex compound characters. The Chinese script is thus an ideographic writing, which does not convert sounds as Latin based languages, but meaning.

4. The culture related color and symbol perception should be included into the general concept.

Since the topic is so complex, the decision here will illustrate only the fourth criteria, color. Because of differing hardware and software capabilities of computers' colors on web sites are not necessarily consistent from machine to machine. Those machine differences could effect "Web Colors", Eye Ailments" (i.e. color deficiency and color blindness), "Blue Effect" (as increasing insensitiveness to the color blue by older people), "3D Effect" (perceived where one color floats above the other, seen between any two colors that are far apart on the visible spectrum and might cause headache) and "Background Color effects" (for the grade of design elements dominance).

The Chinese understanding of colors deviates from the western understanding especially in following fundamental symbolism:

In 3000 B.C. the Chinese assigned colors to the seasons, wind directions, recurring daily segments, and to the human internal organs. Today colors still have emotional and physical characteristics for the Chinese, like "black" for Power, Money, Meditation, Dull Ambience; "white” for Death, Mourning, "yellow” for Tolerance, Patience and Wisdom, and "gold" stands for Power. 
Besides keeping traditional color perceptions in mind, it is also important to understand modern adaptations. For example, in everyday language the Chinese word "hu1ng" for the color yellow also symbolizes pornography. It is interesting to see that the color perception of Chinese and Western Europeans is extremely diverse concerning black, white, and yellow.

\section{Relevance of Eye Tracking}

Eye tracking is a method used to verify where a person is looking. The eye tracker provides objective and quantitative evidence of the user's visual process. Eye movements are generally recorded to determine a user's attention patterns concerning a given stimulus. (Duchowski, 2003, pages 20-26)

Eye tracking cameras can (User Interface Engineering, Website):

- Tell whether users are looking at the screen. Without an eye tracker, it is difficult to verify exactly where users are looking.

- Tell whether users are reading or scanning. It is easy to distinguish reading, a user's systematic fixation on word clusters, from scanning for particular words or phrases.

- $\quad$ Measure the relative intensity of a user's attention to different parts of a web site. By dividing the screen into areas of interest (AOI), such as the top navigation bar or pictures, one can see when and how long a user looks at each AOI.

- Compare scan patterns of all users. By counting how long each user looked at each area, and in what order, scan patterns of groups of users can be compared.

The raw eye movement and pupil diameter data together with the actual gaze position are displayed on the test monitor and can be synchronized with external stimuli and recorded to file. The user interface includes parallel, live video displays of the eye and scene video with online data plots and all required user controls. (We site: SMI iView X System Manual, 2003 and SensoMotoric Instruments)

It is important to remember that an eye tracking device can give an insight of a user's actual perception, but only in conjunction with an adjacent questioning of participants. Just because the eye looks at something does not automatically mean that it is consciously perceived by the brain. It is therefore necessary to question the participants in order to find out what the users remember seeing.

\section{Research Design}

To gain more knowledge about the needs, preferences and behavioral patterns of Chinese Internet users, the experiment was based on the overall hypothesis that web site perception and recognition is different between Chinese and German users, mainly due to cultural differences.

Throughout the experiment, 60 participants were tested while 42 provided valid data. The experimental group consisted of 21 participants from China between 19 and 31 years old. The control group consisted of 21 German users between 19 and 30 years old. In both the German and the Chinese groups, 85.7\% (18 out of 21) stated that they often surf the Internet.

Chinese web sites in Mandarin Chinese were shown to Chinese users and the equivalent web sites in English to German users with an almost identical screen layout (using the same navigation, design, orientation, and content elements). The web sites used for testing were

- China Eastern, a Chinese airline (http://www.ce-air.com/cea/zh_CN/homepage, 08/10/2003/),

- Mercedes-Benz the automobile company (http://www.mercedes-benz.com.cn/mb_china_vrc, 08/10/2003),

- Sony Ericsson, a telecommunications company (http://www.sonyericsson.com/cn/spg.jsp?page=start, 08/10/2003). 
The experiment was conducted in three steps: (Figure 2)

a) Pretest Questioning: The participants were asked demographic information, their Internet experience and the preferences they have.

b) Observation and Recording: Using the Eye Tracking camera the participants were looking around the three web sites tested (China Eastern, Mercedes-Benz, and Sony Ericsson) for 30 seconds per site without instructions.

c) Post-Test Questioning: Directly after the participants viewed each web site, they were questioned regarding their recognition and liking of the web site.

Figure 2: Experiment Procedure

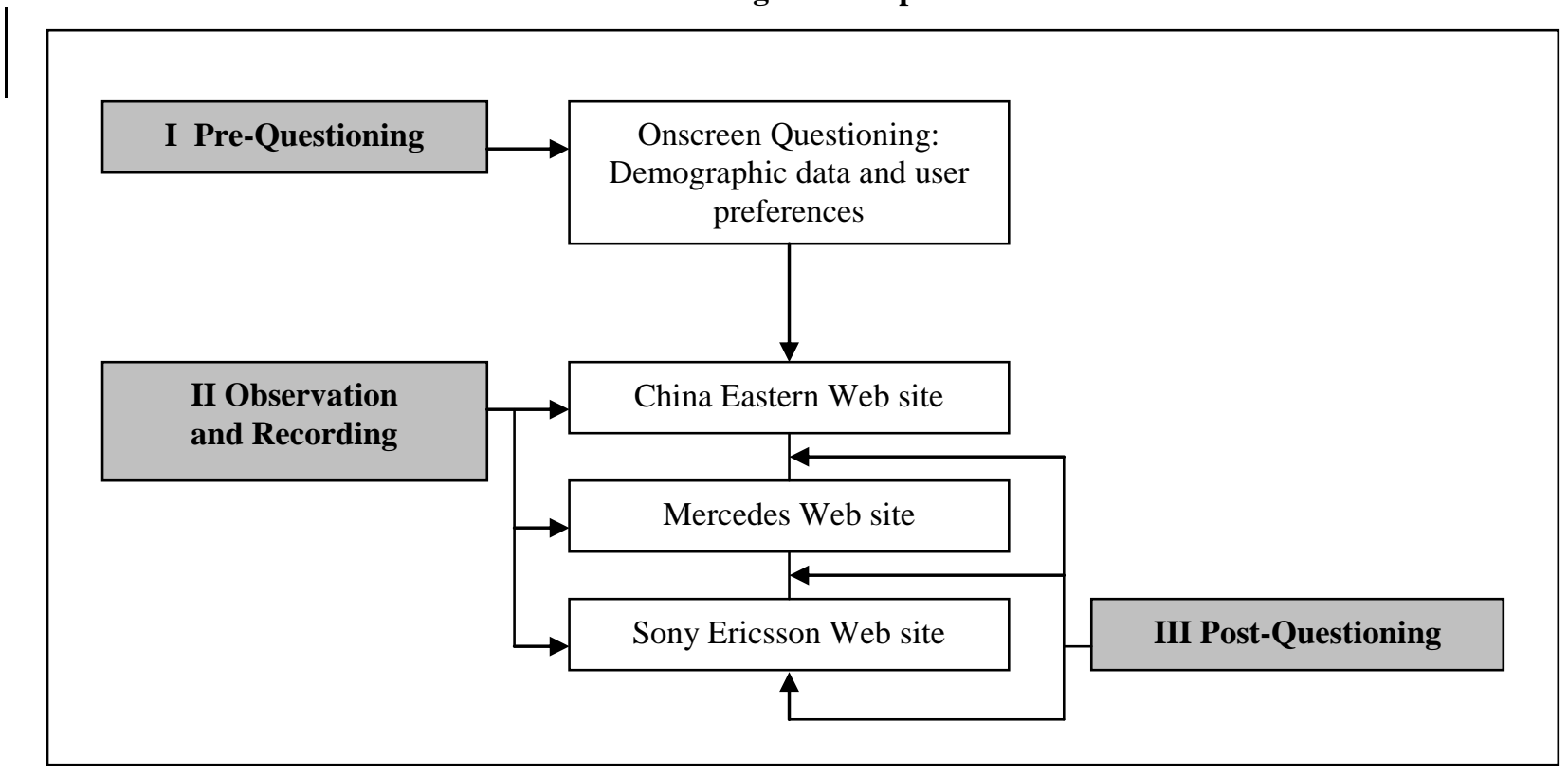

\section{Research Conclusions and Outlook}

The Chinese seem to like search functions on web sites, like graphics and pictures. Only $43 \%$ of the Chinese prefer navigation bars on sites. More than 75 percent of the Germans seem to prefer simple and clear navigation in form of navigation bars, search masks, and hyperlinks within the texts. One-third Germans (no Chinese) who like sitemaps where all experienced user (correlation $r=0.97$ ). All Germans, mainly due to the immense amount of advertisement they contain, disliked pop-up windows. It remained unclear why almost half of the Chinese group favored them.

The participants were also asked to rate the following characteristics for their importance: website design, clarity, color design, terminology, and amount of information, comprehensibility, and degree of up-to-dateness. The rating scale was "important", "somewhat important", "somewhat not important", and "not important". More than 90 percent of the Chinese and German participants rated clarity and comprehensibility as the most important factors of a web site. Chinese rated the amount of information with 95,3 percent and the color design with 86 percent importance; this criteria are 10 to 15 percent higher than for Germans. For Germans, the Design is 95.3 percent important. This is nearly $30 \%$ percent higher than how the Chinese rated this criterion. (Figure 3 ) 
Figure 3: Cultural rating of Website factors

\begin{tabular}{|c|l|l|}
\hline Ranking & \multicolumn{1}{|c|}{ Chinese } & \multicolumn{1}{c|}{ German } \\
\hline $\mathbf{1}$ & Clarity (95.3 \%) & Clarity (95.3\%) \\
\hline $\mathbf{2}$ & Amount of information (95.3\%) & Comprehensibility (95.3\%) \\
\hline $\mathbf{3}$ & Comprehensibility (90.4\%) & Design (95.2 \%) \\
\hline $\mathbf{4}$ & Color design (86\%) & Amount of information (85\%) \\
\hline $\mathbf{5}$ & Up-to-dateness (71.4\%) & Up-to-dateness (81 \%) \\
\hline $\mathbf{6}$ & Design (66\%) & Terminology (81 \%) \\
\hline $\mathbf{7}$ & Terminology (38\%) & Color design (71\%) \\
\hline
\end{tabular}

The Eye Tracking results indicate eight specific findings.

First, concerning the Area of Interest (AOI), results show that Chinese and German participants looked nearly the half of the time at the body text $(37.2 \%)$ and one third at the navigation bar. Pictures and e-commerce functions came next by the Chinese with about 10 percent. The Germans estimated Banner and Logo as next. The eye tracking data revealed that most Germans started reading the text and then abruptly stopped and looked at other design elements. The Chinese that looked at the text read most of it, most likely due to its scannable and abstracted nature.

Second the point of entry (POE), the area where a user looks when first viewing a websit, was researched. Comparing results with the Stanford Poynter Study (Webpage: Stanford Poynter Study) with the conclusion, that users view text nearly twice as often as pictures when entering a website, and the User Interface Engineering Study (Webpage: User Interface Engineering Study) which determined the POE of users to the upper left of the center of the screen, we can only partly confirm the general findings of these studies for all participants. Figure 4 show the different screen locations discussed below. Of all participants, $36.51 \%$ of first views were in the center of the screen (field five) while $31.75 \%$ were in field one. Differences exist when viewing the Chinese and German results separately. While $22.2 \%$ of the Germans had their point of entry in field one, an astounding $41.27 \%$ of Chinese started in this field. The second most common point of entry for Chinese is field 5 with $33.3 \%$ of first views. The Chinese point of entry hierarchy is field 1 and then field 5, which is the exact opposite of the common western order.

With Eye Tracking we could also prove the common knowledge, that the bottom and the right sides of web sites are least looked at.

Figure 4: Chinese and German Point of Entry Allocation

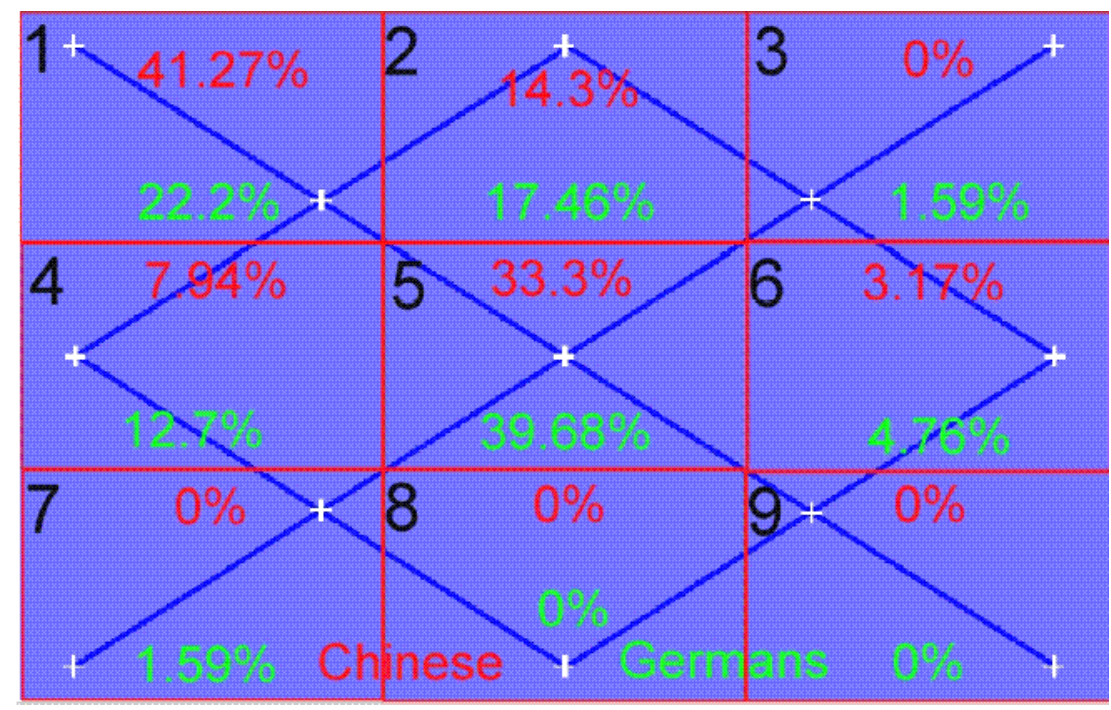


Third: After the point of entry, a user's gaze path is determined by whatever catches their attention. The majority of Germans participants spent less time with reading body text and looking at pictures than Chinese. They were soon focused on navigation elements.

Fourth: Concerning graphics, the Chinese group clearly favored graphics and pictures (81\%) more than the German group $(57.1 \%)$. In average the Chinese participants viewed pictures for a longer period than Germans. But in the post questioning recognition questioning, the German group could clearly state the number of pictures more accurately on all websites than the Chinese group. Both groups perceived graphics and pictures as an informationconveying tool, whereas the results indicate that Chinese also recognize them as a navigation element while Germans perceive them more as an important design feature.

Fifth: The Nielson Netrating study concerning text found that users do not read but scan websites, and that online text must be made scannable. This finding was confirmed by German participants. But most Chinese participants read all of the text and viewed the text AOIs on all three websites for a longer period than Germans during recording. After completion of the experiment, all participants were also asked how they generally liked the websites. Almost all Chinese answered that they thought that the websites did not have enough information.

A pre-study for importance of content on Chinese web sites showed clearly that the Chinese have much content on their web sites. Most Chinese web sites are more focused on transmitting information than on good design. Chinese users are accustomed to full packed web sites and therefore searched the tested sites for more information resulting in higher "other" AOI ratings. These statements are proved by the abovementioned higher Chinese desire for more information and lower design ratings. (Theuner, Steinmetz, 2003)

Sixth: An additional design tool is the use of color. The Chinese participants considered color design as the fourth most important website factor whereas the German group considered it least significant. Astoundingly, recognition of color use in post questioning questioning was clearly dominated by Germans. By rating the colors of the three web sites, Mercedes-Benz web site got the best ratings by Germans whereas the Chinese gave the Sony Ericsson the highest marks. No doubt, using the color silver on the Mercedes-Benz web site is intended to be a modern symbolic of elegance, velocity, and quickness after winning two championships in Formula 1 racing for Europeans. This trend is not the same in other countries. In China, the color silver is neutral and symbolizes metal. Depending on the quality of monitor used, the Mercedes-Benz website sometimes looks more gray than silver. In China gray is the color of self-denial, fear, and depression.

Seventh: The three basic modes of navigation elements are links, a sitemap, and a search engine. The Chinese group, who ranked navigation bars in third place, while they were most important for the Germans, looked at the navigation bars for a much shorter time than the German group (over nine seconds difference between the two groups based on the 30 -second recording time for each participant). Germans also preferred other links such as hyperlinks more than the Chinese did. (Figure 5)

Figure 5: Chinese and German Preferences of Navigation Elements

\begin{tabular}{|l|c|c|}
\hline \multicolumn{1}{|c|}{ Navigationelements } & Chinese & Germans \\
\hline Navigation bars & $42.9 \%$ & $76.2 \%$ \\
\hline Hyperlinks & $28.6 \%$ & $47.6 \%$ \\
\hline Mouse-over/pull-down-menu & $4.8 \%$ & $23.8 \%$ \\
\hline Sitemaps & $4.8 \%$ & $33.3 \%$ \\
\hline Search mask on the website & $61.9 \%$ & $61.9 \%$ \\
\hline Graphics/pictures & $81 \%$ & $57.1 \%$ \\
\hline Pop-up windows & $42.9 \%$ & $0 \%$ \\
\hline doesn't matter & $0 \%$ & $4.8 \%$ \\
\hline (multiple answers possible) & &
\end{tabular}


Eighth: An interesting result of the eye movement recording in context with screen layout was that the less a website "filled" the screen, the more the Chinese eye movements went astray. It is true that the visual tension of a website is not just generated by combining text and pictures, but also by alternating "used" areas on the screen with "empty" areas. The field "other" in the AOI evaluation stands for the time the participants did not look at the other defined AOIs. Generally, the more empty areas the websites had, the more both groups failed to look at AOIs. The Chinese AOI field "other" was always higher than the German.

With Eye Tracking we can confirm findings found in secondary literature, that the logo was viewed longer and its position named more accurately by all participants when located in the conventional top left-hand corner. Sony Erickson placed its logo in the top right-hand corner and viewing time and recognition dropped. After experiment completion, many participants of both groups also stated that the Sony Ericsson logo was too small.

Eye Tracking also showed us some weak points on the tested web sites which were not part of our project. For example: Usability tests have shown that the most common navigation problems are mostly due to overlooked navigation elements. In fact, many participants from both groups stated that the Sony Ericsson website generally used a too small navigation bar and too small font. The eye tracking data revealed that the Sony Ericsson navigation bar was the least viewed of all three websites. The viewing time of navigation bars was 4 times longer by all participants on the China Eastern web site and the Mercedes-Benz web site than on the Sony Ericsson website.

The immense amount of data collected during this study allows us to perform more evaluation. Our further research program contains several milestones in treating this topic as very complex task with observing more users, designing special web sites for special answers, and using statistical methods like multivariate analysis.

Some of the special questions we will study are:

- Whether participants looked at graphics or text first when viewing a web site: Do Chinese also view text before graphics?

- The fixation order of elements: do Chinese and Germans prefer the same order of viewing the elements?

Including other countries, like Japan or some East European countries in this research project will be the next step.

\section{References}

1. Cateora, Philip R.; Graham, John L.: International Marketing; 10th edition, McGraw-Hill, Boston 1999

2. CNNIC Webpage: CNNIC, http://www.cnnic.net.cn/develst/repindex-e.shtml, 8/31/2003

3. Duchowski, Andrew T.: Eye Tracking Methodology, Theory and Practice, Springer Verlag 2003

4. Nielsen//NetRatings: http://www.nielsen-netratings.com/news.jsp?section=dat_to\&country=ge, 11/11/2003

5. Staiger, Brunhild; Friedrich, Stefan; Schütte, Hans-Wilm (Hrsg): Das große China-Lexikon. Geschichte, Geographie, Gesellschaft, Politik, Wirtschaft, Bildung, Wissenschaft, Kultur; Institut für Asienkunde, Primus Verlag, Hamburg 1998

6. Theuner, Gabriele: Erfolgsfaktoren User-orientierter Webseitengestaltung. In :HMD Praxis der Wirtschaftsinformatik, Electronic Business; Heft 215; dpunkt.verlag 2000, pages 69-73

7. User Interface Engineering, http://wrld.std.com/ uieweb/eyetrack2.htm, 08/31/2003

8. SensoMotoric Instruments: - http://www.smi.de/home/index.html, 08/31/2003

9. Stanford University and the Poynter Institute, The Stanford Poynter Project, http://www.poynterextra.org/et/i.htm, 08/31/2003 
Notes 